Journal of Experimental and Clinical Medicine https://dergipark.org.tr/omujecm

Research Article

J Exp Clin Med

2021; 38(4): 599-603

doi: $10.52142 /$ omujecm.38.4.36

\title{
The evaluation of the triglyceride glucose index in symptomatic patients with high suspicion of coronary artery disease: A single center study
}

\author{
Sara ÇETİN ŞANLIALP',* (i), Gökay NAR ${ }^{2}$ (i) \\ ${ }^{1}$ Department of Cardiology, Servergazi State Hospital, Denizli, Turkey \\ ${ }^{2}$ Department of Cardiology, Faculty of Medicine, Pamukkale University, Denizli, Turkey
}

\begin{abstract}
\begin{tabular}{ccccc}
\hline Received: 25.02 .2021 & $\bullet$ & Accepted/Published Online: 07.03.2021 & • & Final Version: 30.08 .2021 \\
\hline
\end{tabular}
\section{Abstract}

Triglyceride glucose index (TyG) plays an important role in metabolic syndrome (MetS), which predisposes to the development of cardiovascular diseases (CVD). Mostly, the association between the TyG index and coronary artery disease (CAD) has been investigated in healthy individuals on long-term follow-up or in asymptomatic patients with a mild and mild-moderate cardiovascular risk profile. However, there are few studies evaluating the association of TyG index with CAD in symptomatic patients. In hence, we aimed to determine the role of the TyG index in the presence and severity of CAD in symptomatic patients with high suspicion of CAD in this study. 100 patients who underwent coronary angiography were included and the patients were divided into two groups according to the presence of CAD. TyG index was calculated by the formula Ln [fasting triglycerides $(\mathrm{mg} / \mathrm{dl})$ fasting glucose $(\mathrm{mg} / \mathrm{dl}) / 2$ ]. The severity of CAD was assessed by angiographic risk scores GENSINI, SYNTAX I, and the number of diseased vessels. TyG index did not differ significantly between patients with and without CAD. Also, there was no significant correlation between TyG index and GENSINI, SYNTAX I, and the number of diseased vessels. (for all p $>0.05$ ) In multiple logistic regression analysis, the only independent predictor of $\mathrm{CAD}$ was age. ( $\mathrm{p}=0.03, \mathrm{OR}=1.048,95 \% \mathrm{CI}=1.004-1.094)$ In conclusion, TyG index may not be a useful marker for predicting the presence or severity of CAD. According to the results of this study, age is the only predictor of CAD independent of all causes.
\end{abstract}

Keywords: coronary artery disease, gensini, syntax i, triglyceride glucose index

\section{Introduction}

Coronary vascular diseases (CVD) are still associated with high mortality and morbidity today, so early diagnosis and appoppriate treatment are important (1). Insulin resistance (IR) is the cornerstone for cardiometabolic diseases, and its increased levels may provide information in diagnosis and progression. Recently, triglyceride glucose index (TyG) derived from fasting blood glucose and triglyceride has been used as an alternative to the hyperinsulinemic-euglycemic clamp (HEC) and homestase model (HOMA-IR) due to its practical use, low cost, high sensitivity and high specificity. (2, 3). Although most of the research with the TyG index has focused on metabolic syndrome (MetS) and MetS related conditions, the cross-sectional studies have found that individuals in high TyG index quartiles are more likely to have arterial stiffness, coronary artery calcification (CAC), carotid atherosclerosis, and stroke than those in the lower quartiles (46). Recently, an increased risk of cardiovascular disease (CVD) has been reported in healthy individuals with a high TyG index (1). In addition, some studies have investigated the effect of TyG index on CVD prognosis (7). However, the importance of the TyG index in symptomatic patients with suspected CAD has not been clearly determined, and to the best of our knowledge, there is no study investigating the severity of CAD by using both SYNTAX I and GENSINI scores in symptomatic patients diagnosed with CAD. In hence in this study, we aimed to investigate the association between TyG index and CAD in symptomatic patients with high suspicion of CAD.

\section{Materials and methods}

\subsection{Study population}

In this retrospective observational case-control study, the medical records of 100 patients who met the inclusion criteria and underwent coronary angiography in our cardiology department between April 2020 and August 2020 were analyzed using hospital database. Inclusion criteria were being 18-90 age intervale, presence of angina or equivalent symptoms and a clinical diagnosis including diagnosis without advanced examination or history of a positive stress test and performing coronary angiography. Cardiomyopathy severe valvular heart disease, previous myocardial infarction or CAD, acute pericarditis or myocarditis, acute or chronic infection, chronic inflammation, malignancy, severe kidney dysfunction and severe liver disease were defined as exclusion criteria. Significant CAD was defined as $50 \%$ or more than $50 \%$ stenosis in at least one epicardial coronary vessel on coronary angiography. This study was consistent with the Helsinki declaration and was approved by our hospital's ethical review 
board (Pamukkale University Faculty of Medicine Hospital, Denizli, Turkey; 2020/20, protocol no:020/70506). All the participants gave an informed consent before enrolling in the study.

\subsection{Data collection}

The basic characteristics of the study population such as age, gender, hypertension, diabetes, current smoking was colllected from medical records. Fasting venous blood samples including blood glucose, lipid parameters, renal functional tests, complete blood counts and, left ventricular ejection fraction (LVEF) calculated by the modified Simpson method were reexamined using hospital database. The TyG index was calculated by the formula $\mathrm{Ln}$ [fasting triglycerides $(\mathrm{mg} / \mathrm{dl}) \mathrm{x}$ fasting glucose (mg / dl) /2] (8). Angiographic data were obtained from cardiac catheterization laboratory records were analyzed randomly by at least two experienced cardiologists who were blind to the study protocol.

\subsection{Coronary lesion severity}

Coronary lesion severity was evaluated by GENSINI score, SYNTAX I score and the number of diseased vessels. The Gensini score was calculated by assigning a severity score to each coronary lesion according to the stenotic degree of the coronary artery lumen and the importance of its localization. The total score was equal to the sum of the stenosis score and the location score for all diseased segments (9) (stenosis of $25 \%, 50 \%, 75 \%, 90 \%, 99 \%$, and total occlusion were given Gensini scores of $1,2,4,8,16$, and 32, respectively and location score was calculated by multiplying by the constant coefficient: left anterior descending coronary artery (LAD) $\times$ 2.5; the proximal segment of the circumflex artery $(\mathrm{LCX}) \times 2.5$; the mid-segment of the $\mathrm{LAD} \times 1.5$; the right coronary artery (RCA), the distal segment of the LAD, the posterolateral artery and the obtuse marginal artery $\times 1$; and others $\times 0.5$ ).

SYNTAX I score was calculated for stenosis diameter of $50 \%$ or greater in vessels of $1.5 \mathrm{~mm}$ or more in diameter and the final online updated version (2.11) was used (10).

\subsection{Statistical analysis}

All data were analyzed using SPSS v.17.0 for Windows (SPSS, Inc., Chicago, Ill., USA). Categorical variables were expressed as frequencies and percentages and continuous variables were presented as means \pm SD. Kolmogorov-Smirnov test was used for determination of normal distribution. In independent groups comparisons, independent samples t test was used when parametric test assumptions were provided and Mann-Whitney $\mathrm{U}$ test was used when parametric test assumptions were not provided. $\chi 2$ test was used for comparison of categorical values. Correlation between variables was done using Pearson's correlation analysis. Multiple logistic regression analysis was performed to determine the risk factors affecting CAD, which is the dependent variable. The threshold of statistical significance was considered as $\mathrm{p}<0.05$.

\section{Results}

The baseline characteristics of the study population are summarized in Table 1. Age, the incidence of hipertension and diabetes, fasting blood glucose differed significantly in patients with and without CAD $(\mathrm{p}<0.05)$. However, no significant difference was found in gender, smoking, LVEF, HbAlc, creatinine, CRP, hemogram, WBC and lipid parameters between groups. The TyG index was calculated as $9.01 \pm 0.49$ and $8.82 \pm 0.46$, respectively, but no statistical difference was observed $(\mathrm{p}=0.064)$. In addition, there was no significant difference in TG/HDL-C and LDL-C/HDL-C values.

The GENSINI and SYNTAX I scores of patients with symptomatic CAD were calculated as $51.74 \pm 31.38$ and $24.42 \pm 10.14$ respectively, and $42 \%$ had single-vessel disease, $28 \%$ had two-vessel disease, and $30 \%$ had multi-vessel disease. The TyG index was correlated with only TG/HDL-C which is another indicator used in IR evaluation. There was no significant correlation between TyG index with CAD severity scores identified by GENSINI, SYNTAX I and the number of diseased vessels (Table 2, Figs. 1-3) In the multiple logistic regression analysis, age was significantly associated with CAD regardless of all causes. ( $\mathrm{p}=0.03, \mathrm{OR}=1.048,95 \% \mathrm{CI}=1.004-$ 1.094) (Table 3).

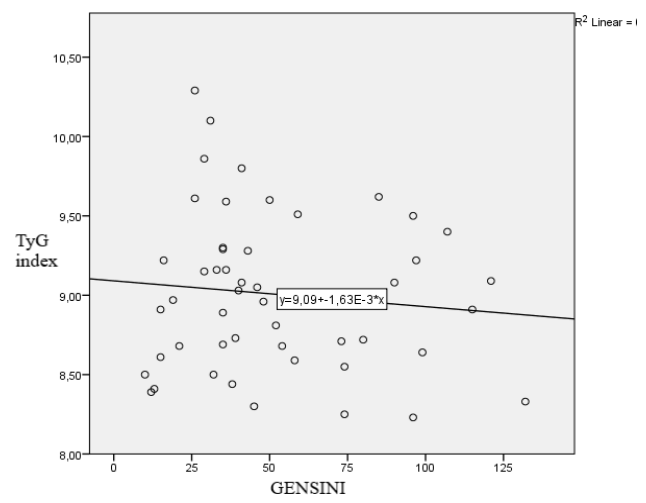

Fig. 1. The correlation between TyG index and GENSINI score. TyG index, triglyceride glucose index

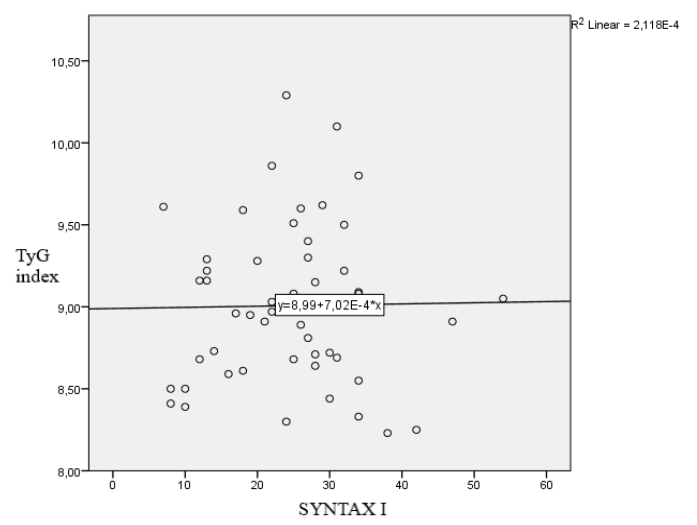

Fig. 2. The correlation between TyG index and SYNTAX I score. TyG index, triglyceride glucose index 
Sanlialp and Nar / J Exp Clin Med

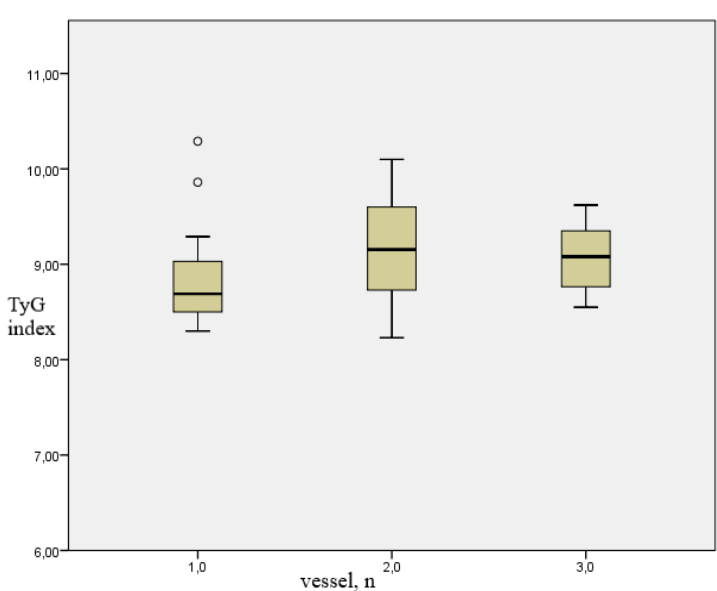

Fig. 3. The correlation between TyG index and the number of diseased vessels. TyG index, triglyceride glucose index

Table 1. Baseline characteristics of study population

\begin{tabular}{|c|c|c|c|}
\hline Variables & $\begin{array}{c}\text { Patients with } \\
\text { CAD } \\
(\mathrm{n}=50)\end{array}$ & $\begin{array}{c}\text { Patients without } \\
\text { CAD } \\
(\mathrm{n}=50)\end{array}$ & $\mathrm{p}$ \\
\hline Mean age (years) & $63.80 \pm 10.63$ & $57.56 \pm 13.37$ & 0.016 \\
\hline Males, n (\%) & $37(74)$ & $31(62)$ & 0.209 \\
\hline $\begin{array}{l}\text { Hypertension, } \mathrm{n} \\
(\%)\end{array}$ & $34(68)$ & $23(46)$ & 0.038 \\
\hline $\begin{array}{l}\text { Diabetes mellitus, } n \\
(\%)\end{array}$ & $22(44)$ & $11(22)$ & 0.020 \\
\hline $\begin{array}{l}\text { Current smoking, } n \\
(\%)\end{array}$ & $13(26)$ & $17(34)$ & 0.450 \\
\hline LVEF (\%) & $54.24 \pm 9.58$ & $53.79 \pm 8.99$ & 0.823 \\
\hline 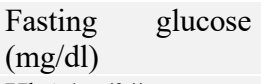 & $130.60 \pm 51.04$ & $111.72 \pm 29.24$ & 0.042 \\
\hline $\mathrm{HbAlc}(\%)$ & $8.64 \pm 2.04$ & $8.10 \pm 1.23$ & 0.623 \\
\hline Creatinine (mg/dl) & $1.01 \pm 0.30$ & $1.00 \pm 0.58$ & 0.239 \\
\hline TChol (mg/dl) & $178.44 \pm 41.21$ & $176.56 \pm 40.87$ & 0.831 \\
\hline LDL-C (mg/dl) & $108.56 \pm 37.06$ & $105.00 \pm 36.32$ & 0.651 \\
\hline HDL-C (mg/dl) & $40.82 \pm 10.80$ & $43.90 \pm 11.78$ & 0.203 \\
\hline $\mathrm{TG}(\mathrm{mg} / \mathrm{dl})$ & $145.22 \pm 64.04$ & $133.59 \pm 46.51$ & 0.342 \\
\hline Hemoglobin (g/dl) & $13.28 \pm 1.97$ & $15.63 \pm 2.95$ & 0.209 \\
\hline $\mathrm{WBC}($ cells $/ \mu \mathrm{L}))$ & $9.60 \pm 2.76$ & $9.23 \pm 2.95$ & 0.543 \\
\hline CRP (mg/dl) & $1.35 \pm 3.14$ & $3.20 \pm 5.55$ & 0.060 \\
\hline TyG index & $9.01 \pm 0.49$ & $8.82 \pm 0.46$ & 0.064 \\
\hline TG/HDL-C & $3.82 \pm 2.07$ & $3.35 \pm 1.76$ & 0.260 \\
\hline LDL-C/HDL-C & $2.82 \pm 1.18$ & $2.47 \pm 0.82$ & 0.126 \\
\hline GENSINI & $51.74 \pm 31.38$ & & \\
\hline SYNTAX I & $24.42 \pm 10.14$ & & \\
\hline $\begin{array}{l}\text { Single- } \\
\text { disease }\end{array}$ & $21(42)$ & & \\
\hline Two-vessel disease & $14(28)$ & & \\
\hline Multivessel disease & $15(30)$ & & \\
\hline
\end{tabular}

LVEF, left ventricular ejection fraction; TChol; total cholesterol; LDL-C, low-density lipoprotein cholesterol; HDL-C, high-density lipoprotein cholesterol; TG, triglycerides; WBC, white blood cells; CRP, C-reactive protein; TyG index, triglyceride- glucose index; CAD, coronary artery disease

\section{Discussion}

In this study, we investigated the role of the TyG index in determining presence and severity of CAD in symptomatic patients with high suspicion of CAD. We found that TyG index did not predict neither CAD severity nor CAD presence in this population. The previously studies have shown the association of TyG index with CAD related conditions such as diabetes, hypertension and MetS. For these reasons, the TyG index has been claimed as an atherogenic marker $(2,11)$.

Table 2. Correlation analysis

\begin{tabular}{|l|c|c|}
\hline TyG index & $\mathrm{r}$ & $\mathrm{p}$ \\
\hline Mean age & 0.031 & 0.773 \\
\hline LVEF & 0.025 & 0.809 \\
\hline Fasting glucose & 0.602 & $<0.001$ \\
\hline HbAlc & 0.183 & 0.465 \\
\hline Creatinine & -0.054 & 0.611 \\
\hline Tchol & 0.075 & 0.481 \\
\hline LDL-C & -0.080 & 0.453 \\
\hline HDL-C & -0.158 & 0.137 \\
\hline TG & 0.751 & $<0.001$ \\
\hline Hemoglobin & -0.113 & 0.289 \\
\hline WBC & 0.167 & 0.116 \\
\hline CRP & 0.086 & 0.417 \\
\hline TG/HDL-C & 0.687 & $<0.001$ \\
\hline LDL-C/HDL-C & 0.078 & 0.465 \\
\hline GENSINI & -0.104 & 0.470 \\
\hline SYNTAX I & 0.014 & 0.920 \\
\hline Stenotic vesssel & 0.181 & 0.251 \\
\hline numbers & & \\
\hline
\end{tabular}

LVEF, left ventricular ejection fraction; TChol; total cholesterol; LDL-C, low-density lipoprotein cholesterol; HDL-C, high-density lipoprotein cholesterol; TG, triglycerides; WBC, white blood cells; CRP, C-reactive protein; TyG index, triglyceride glucose index.

Table 3. Factors that independently correlated with the CAD in multiple logistic regression analysis

\begin{tabular}{|l|l|l|l}
\hline Variable & $\mathbf{P}$ & OR & $\mathbf{9 5 \% C I}$ \\
\hline Mean age & 0.031 & 1.048 & $1.004-1.094$ \\
Gender & 0.121 & 2.426 & $0.792-7.436$ \\
Diabetes & 0.486 & 1.005 & $0.991-1.019$ \\
Hipertension & 0.605 & 1.303 & $0.477-3.560$ \\
TyG index & 0.556 & 1.445 & $0.424-4.925$
\end{tabular}

$\mathrm{CAD}$, coronary artery disease; TyG index, triglyceride glucose index; OR, odd ratio; CI, confidence interval

In our study, the correlation between the TyG index and the TG / HDL-C, which is also one of the IR indicators, confirms that the TyG index may be used in evaluation of dysmetabolic conditions. Recently, the studies have been reported the effects of the TyG index on atherosclerosis. In one study, increased TyG index predicted the adverse cardiovascular event in healty population (12). Also, the TyG index was associated with arterial stiffness and the TyG index showed the CAC presence on computer tomography imagings in healty individuals with no cardiovascular risk factors in other studies $(13,14)$. In addition, a study has confirmed that the TyG index is associated with carotid atherosclerosis and arterial stiffness in lean postmenopausal women and another study showed that TyG index was increased in asymptomatic diabetic patients with coronary artery stenosis $(15,16)$. Moreover, one study has suggested that TyG index can be used in early detection of subclinical CAD in the absence of traditional CV risk factors (17). However, unlike these studies, we failed to show the relationship between the TyG index and the presence of CAD in our study. But these studies had some limitations such as performing with healthy population or using postmenopausal woman or including specific study population such as diabetic patients. Also, the individuals were asymtomatical in these 
studies. However, we included symptomatic patients with high suspicion of CAD and with various cardiovascular risk factors in our study. As if to support our work, one study showed that high TyG index may indicate CAD risk profile only in healthy subjects, not patients with cardiovascular risk factors such as hipertension or diabetes and another study demonstrated that the TyG index may be an indicator for type 2 diabetes rather than CAD $(18,19)$. Inconsistencies in studies investigating the relationship between the TyG index and CAD may be linked to the the properties of study population, age distribution, ethnic diversity, the cardiovascular risk profile, and duration of exposure to dysmetobolic conditions. Also, providing glucose and lipid regulation by using antihyperlipidemic and antidiabetic drugs may weaken or eliminate this relationship. Indeed, Silvia et al reported that the TyG index was higher in patients with at least one cardiovascular risk factor and cardiac symptoms than in patients with known CAD and under treatment (20).

Recently, researchers have focused on the effect of the TyG index on atherosclerosis progression not only presence. A study involving prediabetic patients with acute coronary syndrome (ACS), there was a positive correlation between SYNTAX I and TyG index (21). In another study, Mao et al. showed that the patients with increased TyG index had high SYNTAX I scores in non-ST elevation miyocardial infarction (2). In addition, asymptomatic diabetic patients with high TyG index had severe CAD identified by stenotic degree and the number of diseased vessels during scanning with computer tomography in another study (22). But we could not find any relationship between the TyG index with GENSINI, SYNTAX I and number of diseased vessels unlike these studies. Also, these studies were performed in patients with ACS or asymptomatical patients and the patients using statins or fibrates were not included. Unlike these studies, Alizargar et al suggested that TyG index may be used in early stage of atherosclerosis and that TyG index is associated with presence of atherosclerosis not severity (23). In another study, Won et al. could not show the association of the TyG index with CAC progression in patients with heavy calcium burden (24). These studies findings support our results. Moreover, dynamic changes in the TyG index or prolonged exposure to cardiovascular risk factors may provide more accurately estimation of CAD severity than high TyG index.

The age was only independent predictor of CAD in our study and most studies have shown that age continues to be an independent predictor for CAD even if traditional risk factors are removed by using advanced analysis methods (25). This may be explained by the prolonged exposure to cardiovascular risks, higher cardiovascular burden and comorbidity increase with age.

There were some limitations in our study. The study was designed as retrospectively. Our study population was relatively small, and it was performed in a single center.
Therefore, the study results may not be generalized to the whole population. Also, triglyceride and fasting glucose were only mesured at baseline, body mass index or waist circumference and dietary habits were not recorded. In addition, we could not ignore drug use that could affect the results because of retrospective study design.

In conclusion, the use of TyG index in determining CAD severity or presence may not be aproppriate in symptomatic patients with high suspicion of CAD and the age is the only independent predictor in CAD according to our results. However multi-center prospective studies are needed to evaluate the role of the TyG index in CAD more accurately.

\section{Conflict of interest}

None to declare.

\section{Acknowledgments}

None to declare.

\section{References}

1. Park GM, Cho YR, Won KB, Yang YJ, Park S, Ann SH, et al. Triglyceride glucose index is a useful marker for predicting subclinical coronary artery disease in the absence of traditional risk factors. Lipids Health Dis. 2020 Jan 14;19(1):7.

2. Mao Q, Zhou D, Li Y, Wang Y, Xu SC, Zhao XH. The triglyceride-glucose index predicts coronary artery disease severity and cardiovascular outcomes in patients with non-ST segment elevation acute coronary syndrome. Dis. Markers. $2019 ; 6891537$.

3. Kim JH, Lee DY, Park SE, Park CY, Lee WY, Oh KW, et al. Triglyceride glucose index predicts coronary artery calcification better than other indices of insulin resistance in Korean adults: the Kangbuk Samsung Health Study. Precis. Future Med. 2017; 1, 43-51.

4. Jin JL, Cao YX, Wu LG, You XD, Guo YL, Wu NQ, et al. Triglyceride glucose index for predicting cardiovascular outcomes in patients with coronary artery disease. J Thorac Dis. 2018; 10, 6137-6146.

5. Irace $\mathrm{C}$, Carallo $\mathrm{C}$, Scavelli FB, De Franceschi MS, Esposito $\mathrm{T}$, Tripolino C, et al. Markers of insulin resistance and carotid atherosclerosis. A comparison of the homeostasis model assessment and triglyceride glucose index. Int J Clin Pract. 2013; 67, 665-72.

6. Sánchez-Iñigo L, Navarro-González D, Fernández-Montero A, Pastrana-Delgado J, Martínez JA, Risk of incident ischemic stroke according to the metabolic health and obesity states in the Vascular-Metabolic CUN cohort. Int J Stroke. 2017; 12, 187-91.

7. Zhao Q, Zhang TY, Cheng YJ, Ma Y, Xu YK, Yang JQ, et al. Impacts of triglyceride glucose index on prognosis of patients with type 2 diabetes mellitus and non-ST segment elevation acute coronary syndrome: results from an observational cohort study in China. Cardiovasc Diabetol. 2020;19, 108.

8. Simental-Mendía LE, Rodríguez-Morán M, Guerrero-Romero F, The product of fasting glucose and triglycerides as surrogate for identifying insulin resistance in apparently healthy subjects. Metab Syndr Relat Disord 2008; 6, 299-304.

9. Gensini GG. A more meaningful scoring system for determining the severity of coronary heart disease. Am J Cardiol. 1983; 51, 606.

10. SYNTAX working group. SYNTAX score calculator. Available at http://www.syntaxscore.com. Accessed May 20, 2012. 


\section{Sanlialp and Nar / J Exp Clin Med}

11. Ormazabal V, Nair S, Elfeky O, Aguayo C, Salomon C, Zuñiga FA. Association between insulin resistance and the development of cardiovascular disease. Cardiovasc Diabetol 2018; 17, 122.

12. Sánchez-Iñigo L, Navarro-González D, Fernández-Montero A, Pastrana-Delgado J, Martínez JA. Risk of incident ischemic stroke according to the metabolic health and obesity states in the Vascular-Metabolic CUN cohort. 2017; Int J Stroke. 12, 187-91.

13. Lee EY, Yang HK, Lee J, Kang B, Yang Y, Lee SH, et al. Triglyceride glucose index, a marker of insulin resistance, is associated with coronary artery stenosis in asymptomatic subjects with type 2 diabetes. Lipids Health Dis. 2016; 15, 155.

14. Kim MK, Ahn CW, Kang S, Nam JS, Kim KR, Park, JS. Relationship between the triglyceride glucose index and coronary artery calcification in Korean adults. Cardiovasc. Diabetol. 2017; 16, 108.

15. Lambrinoudaki I, Kazani MV, Armeni E, Georgiopoulos G, Tampakis K, Rizos D, et al. The TyG index as a marker of subclinical atherosclerosis and arterial stiffness in lean and overweight postmenopausal women. Heart Lung Circ. 2018; 27 , 716-24

16. Lee SB, Ahn CW, Lee BK, Kang S, Nam JS, You JH, et al. Association between triglyceride glucose index and arterial stiffness in Korean adults. Cardiovasc. Diabetol. 2018; 17, 41.

17. Park GM, Cho YR, Won KB, Yang YJ, Park S, Ann SH, et al. Triglyceride glucose index is a useful marker for predicting subclinical coronary artery disease in the absence of traditional risk factors. Lipids Health Dis. 2020; 19, 7.

18. Cho YR, Ann SH, Won KB, Park GM, Kim YG, Yang DH, et al. Association between insulin resistance, hyperglycemia, and coronary artery disease according to the presence of diabetes.
Sci. Rep. 2019; 9, 6129

19. Vega GL, Barlow, CE, Grundy SM, Leonard D, De Fina LF. Triglyceride-to-high-density-lipoprotein-cholesterol ratio is an index of heart disease mortality and of incidence of type 2 diabetes mellitus in men. J. Investig. Med. 2014; 62, 345-349.

20. da Silva A, Caldas APS, Hermsdorff HHM, Bersch-Ferreira ÂC, Torreglosa CR, Weber B, et al. Triglyceride-glucose index is associated with sympto- matic coronary artery disease in patients in secondary care. Cardiovasc. Diabetol. 2019; 18, 89.

21. Sulistyono $T$, Nughara $T$, Wasyanto $T$. Glucose trigliserdie index as a predictor of severity of coronary artery assesesed with syntax score I in ac1uye coronary sydrome patients. ACI. 2020; $6,127-134$

22. Thai PV, Tien HA, Minh H, Valensi P. Triglyceride glucose index for the detection of asymptomatic coronary artery stenosis in patients with type 2 diabetes Cardiovasc. Diabetol. 2020; 19, 137.

23. Alizargar J, Bai CH. Comparison of carotid ultrasound indices and the triglyceride glucose index in hypertensive and normotensive community-dwelling individuals: a case control study for evaluating atherosclerosis. Medicina (Kaunas, Lithuania). 2018; 54, 71 .

24. Won KB, Park EJ, Han D, Lee JH, Choi SY, Chun EJ, et al. Triglyceride glucose index is an independent predictor for the progression of coronary artery calcication in the absence of heavy coronary artery calcication at baseline. Cardiovasc. 2020; Diabetol.19, 34

25. Dhingra R, Vasan RS. Age as a Cardiovascular Risk Factor. Med Clin North Am. 2002; 96, 87-91. 